\title{
REVIEW
}

\section{Milk oligosaccharides: A review}

\author{
DIANA L OLIVEIRA, ${ }^{1,2}$ R ANDREW WILBEY, ${ }^{1}$ ALISTAIR S \\ GRANDISON $^{1}$ and LUÍSA B ROSEIRO ${ }^{2} *$ \\ ${ }^{1}$ Department of Food and Nutritional Sciences, University of Reading, Whiteknights Reading RG6 6AP, UK, and \\ ${ }^{2}$ Laboratório Nacional de Energia e Geologia (LNEG), Unidade de Bioenergia, Edifício K2, Estrada do Paço do \\ Lumiar 22, Lisboa 1649-036, Portugal
}

Milk oligosaccharides (OSs) confer unique health benefits to the neonate. Although human digestive enzymes cannot degrade these sugars, they support specific commensal microbes and act as decoys to prevent the adhesion of pathogenic micro-organisms to gastrointestinal cells. The limited availability of human milk oligosaccharides (HMOs) impedes research into these molecules and their potential applications in functional food formulations. Recent studies show that complex OSs with fucose and $\mathrm{N}$-acetyl neuraminic acid (key structural elements of HMO bioactivity) also exist in caprine milk, suggesting a potential source of bioactive milk OSs suitable as a functional food ingredient.

Keywords Carbohydrates, Prebiotics, Caprine milk.

*Author for correspondence. E-mail: luisa.roseiro@lneg.pt

(C) 2015 Society of Dairy Technology

\section{INTRODUCTION}

The significant contribution of dairy components as physiologically functional foods was first recognised by the Japanese in the 1980s. At that time, functional food was defined as 'any food ingredient that may provide a health benefit beyond the traditional nutrients that it contains' (Macfarlane et al. 2006). During the last two decades, functional foods have received increasing attention (Bhat and Bhat 2010), particularly in respect of positive effects on host health and/ or well-being beyond their nutritional value (Bhat and Bhat 2011). Milk can be considered the model 'nutraceutical', that is food that conveys immunological and other health benefits together with the nutritional contribution. Significant protection of babies by feeding human milk has been demonstrated in relation to diarrhoeal diseases, respiratory tract infections, bacteraemia and meningitis (Morrow et al. 2005). For over 30 years, it has been accepted that breastfed infants are better protected against infectious agents than formula-fed infants, attributable to the evolution of milks to fit the needs of that species.

Various factors present in milk are known to modulate the developing microbiota within the infant gastrointestinal tract (GIT), including immunoglobulins, lactoferrin, lysozyme, bioactive lipids, leucocytes and various milk glycans (glycolipids, glycoproteins and free oligosaccharides) (Newburg et al. 2005). Of the functional ingredients, oligosaccharides (OSs) are arguably the most important, as they function as prebiotics.

\section{PREBIOTICS}

Prebiotics are defined as 'a selectively fermented ingredient that allows specific changes, in both the composition and/or activity in the gastrointestinal microflora that confers benefits upon host well-being and health' (Gibson et al. 2004; Roberfroid 2007a). This definition requires that prebiotics are resistant to gastric acidity, hydrolysis by host enzymes and gastrointestinal absorption.

Others define prebiotics as 'a carbohydrate that results in changes in numbers of key bacterial genera in the colon, for example bifidobacteria' (Palframan et al. 2003). The latter definition not only considers the microflora changes in the colonic ecosystem of humans but also in the whole GIT and, as such, extrapolates the definition into other areas that may benefit from a selective targeting of particular micro-organisms. Macfarlane et al. (2006) stated that prebiotics are short-chain carbohydrates that have unusual 